Pacific Journal of Mathematics

THE POINTS OF LOCAL NONCONVEXITY OF STARSHAPED 


\title{
THE POINTS OF LOCAL NONCONVEXITY OF STARSHAPED SETS
}

\section{Fausto A. Toranzos}

\begin{abstract}
The notion of point of local nonconvexity has been an important tool in the study of the geometry of nonconvex sets, since Tietze characterized, more than fifty years ago, the convex subsets of $E^{n}$ as those connected sets without points of local nonconvexity. It is proved here that for each convex component $K$ of a closed connected set $S$ in a locally convex space there exist points of local nonconvexity of $S$ arbitrarily close to $K$, unless $S$ itself be convex. Klee's generalization of the just quoted Tietze's theorem follows immediately. The notion of "higher visibility" is introduced in the last section, and three Krasnosselsky-type theorems involving the points of local nonconvexity are proved.
\end{abstract}

1. Notations and basic definitions. The interior, closure, boundary and convex hull of a set $S$ are denoted by int $S$, cl $S$, bdry $S$ and conv $S$, respectively. The closed segment joining $x$ and $y$ is denoted [xy]. If $x \in S$ and $y \in S$, we say that $x$ sees $y$ via $S$ if $[x y] \subset S$. The star of $x$ with respect to $S$ is the set $s t(x ; S)$ of all points of $S$ that see $x$ via $S$. A star-center of $S$ is a point $x \in S$ such that $s t(x ; S)=S$, that is a point of $S$ that sees the whole $S$. The kernel of $S$ is the set ker $S$ of all the star-centers of $S . \quad S$ is starshaped if $\operatorname{ker} S \neq \varnothing$. A convex component of $S$ is a maximal convex subset of $S$. The point $x \in$ bdry $S$ is a point of local nonconvexity of $S$ if for every neighborhood $U$ of $x$, the set $U^{\prime}=U \cap S$ is not convex. The set of all points of local nonconvexity of $S$ is denoted lnc $S$. The origin (null-vector) of a linear topological space is denoted by $\theta$, and the family of its neighborhoods by $\mathscr{N}(\theta)$.

\section{Points of local nonconvexity and convex components.}

THEOREM 2.1. Let $S$ be a closed connected nonconvex set in a locally convex linear topological space, and $K$ be a convex component of $S$. Then $(K+V) \cap \operatorname{lnc} S \neq \varnothing$ for each $V \in \mathscr{N}(\theta)$.

Proof. It is clear that $K$ is closed and, a fortiori, closed in the relative topology of $S$. Assume there exists a $V \in \mathscr{N}(\theta)$ such that $(K+V) \cap \operatorname{lnc} S=\varnothing$. If $x \in K$ there must exist a $U \in \mathscr{N}(\theta)$ such that $U \subset V$ and $U^{\prime}=(U+x) \cap S$ be convex. We intend to prove that $U^{\prime} \subset K$. On the contrary, suppose there exist $y \in U^{\prime}$ and $z \in K$ such that $y$ does not see $z$ via $S$. Let $y_{0}$ be the last point of [xz] 
(going from $x$ to $z$ ) that is visible from $y$. By Lemma 1 of [7], it is easy to verify that $\left[y y_{0}\right] \cap$ bdry $S$ would contain a point $p \in \operatorname{lnc} S$. But then $p \in K+U \subset K+V$, in contradiction with our basic assumption. Hence there are no such points $y$ and $z$. That is $\forall y \in U^{\prime}$ and $\forall z \in K,[y z] \subset S$. This implies that $\operatorname{conv}\left(U^{\prime} \cup K\right) \subset S$, and by the maximality of $K, \operatorname{conv}\left(U^{\prime} \cup K\right)=K$ and $U^{\prime} \subset K$. Since this is true for every $x \in K, K$ is open in the relative topology of $S$. The connectedness of $S$ implies that $K=S$, a fact that contradicts the nonconvexity of $S$. Hence no such $V$ can exist.

We are tempted to substitute the thesis of 2.1 by the stronger statement " $K \cap \operatorname{lnc} S \neq \varnothing$ ". Unfortunately this is false, as the following counterexample shows. If we define $S=\left\{\left.(x ; y) \in R^{2}|y \leqq| x\right|^{-1}\right\}$ then $K=\{(x ; y) \mid y \leqq 0\}$ is a convex component of $S$ but $K \cap \operatorname{lnc} S=$ $\varnothing$. The next corollary considers a situation where this stronger statement holds.

COROLlaRY 2.2. Let $S$ be a closed connected set in a locally convex linear topological space such that $\operatorname{lnc} S$ be compact or empty, and let $K$ be a convex component of $S$. Then the following statements are equivalent: (i) $K=S$ (ii) $K \cap \operatorname{lnc} S=\varnothing$.

Proof. Clearly (i) implies (ii). On the other hand, assume that $K \cap \operatorname{lnc} S=\varnothing$. We intend to prove the existence of a neighborhood $V_{0}$ of $\theta$ such that $\left(K+V_{0}\right) \cap \operatorname{lnc} S=\varnothing$. The inexistence of a neighborhood would allow us to pick a net $\left\{t_{V}, V \in \mathscr{N}(\theta)\right\}$ in $\operatorname{lnc} S$ such that for every $V \in \mathscr{N}(\theta) t_{V} \in(K+V) \cap \operatorname{lnc} S$. The compactness of lnc $S$ would imply the existence of a converging subnet, which in turn would contradict (ii). Hence the existence of $V_{0}$ is proved, in contradiction with the thesis of the previous theorem. Then $S$ must be convex and (i) holds.

We conclude this section with a new proof for the classical Tietze-Klee theorem, originally stated in [3].

THEOREM 2.3 (Tietze-Klee). Let $S$ be a closed connected set in a locally convex linear topological space. Then the following statements are equivalent: (i) $\operatorname{lnc} S=\varnothing$. (ii) $S$ is convex.

Proof. It is clear that (ii) implies (i). On the other hand, (i) contradicts the conclusion of Theorem 2.1. Hence $S$ must be convex.

3. Three Krasnosselsky-type theorems. The point $p$ has higher visibility via $S$ than the point $q$ if $\operatorname{st}(p ; S) \supset \operatorname{st}(q ; S)$. The relation 
"has higher visibility via $S$ than" is a partial ordering in $S$, and the star-centers of $S$ (if there exist such points) should be the maximal elements for this ordering. The visibility cell of $p$ is the set $\operatorname{vis}(p)$ of all the points of $S$ having higher visibility via $S$ than $p$. Of course, $p \in \operatorname{vis}(p)$ always.

LEMMA 3.1. The visibility cell of $p$ is the intersection of all the convex components of $S$ that include $p$.

Proof. Let $x \in \operatorname{vis}(p)$ and $K$ be a convex component of $S$ that includes $p$. Then $K \subset \operatorname{st}(p ; S) \subset s t(x ; S)$. This inclusion implies that $K^{\prime}=\operatorname{conv}(\{x\} \cup K) \subset S$, and the maximality of $K$ yields $K=K^{\prime}$. Hence $x \in K$. Conversely, let $x$ belong to the intersection of all the convex components of $S$ that include $p$, and let $z \in \operatorname{st}(p ; S)$. There is a convex component $K_{0}$ of $S$ such that $[z p] \subset K_{0}$. But $x \in K_{0}$ by construction. Hence $x$ sees $z$ via $S$. Since the argument holds for each $z \in \operatorname{st}(p ; S), x \in \operatorname{vis}(p)$.

It is important to observe that the preceding characterization of $\operatorname{vis}(p)$ uses no topological structure whatsoever.

THEOREM 3.2. Let $S$ be a closed connected nonconvex set in a locally convex linear topological space, such that $\operatorname{lnc} S$ be compact. The kernel of $S$ is the intersection of the visibility cells of all its points of local nonconvexity.

Proof. Let $A=\cap\{\operatorname{vis}(p) \mid p \in \operatorname{lnc} S\}$. Corollary 2.2 and Lemma 3.1 imply that $A$ is the intersection of all the convex components of $S$. Whence, by the lemma that precedes Theorem 2 of [6], $A=$ ker $S$.

Three well-known theorems concerning intersections of families of convex sets are quoted here for later reference.

THEOREM 3.3 (Helly [2]). Let $\mathscr{K}$ be a collection of compact convex sets in $E^{n}$, containing at least $n+1$ members, and such that each subfamily of $n+1$ members have nonempty intersection. Then, the intersection of all the members of $\mathscr{K}$ is not empty.

THEOREM 3.4 (Klee [4]). Let $\mathscr{K}$ be a collection of compact a convex sets in $E^{n}$, containing at least $n+1$ members, and let $C$ be compact convex set in $E^{n}$ such that for each subfamily of $n+1$ members of $\mathscr{K}$ there exists a translate of $C$ included in the intersection of the subfamily. Then, there exists a translate of $C$ included 
in the intersection of all the collection $\mathscr{K}$.

THEOREM 3.5 (Grünbaum [1]). Let $n$ and $k$ be integers such that $n \geqq k>0$, and let $h(n ; k)$ be defined by: (i) $h(n ; n)=n+1$ (ii) $h(n ; 1)=2 n$ (iii) $h(n ; k)=2 n-k$ for $n>k>1$. Let $\mathscr{K}$ be a finite collection of convex sets in $E^{n}$ containing at least $h(n ; k)$ members, and such that each subfamily of $h(h ; k)$ members has intersection of dimension at least $k$. Then the intersection of all the collection $\mathscr{K}$ is of dimension at least $k$.

THEOREM 3.6. Let $S$ be a compact connected nonconvex set in $E^{n}$ such that for every $k$-pointed subset $\left\{t_{1} ; \cdots ; t_{k}\right\}$ of $\operatorname{lnc} S$, with $k \leqq$ $n+1$ there exists a point having higher visibility via $S$ than each $t_{i}$. Then $S$ is starshaped.

Proof. Consider the family $\mathscr{K}=\{\operatorname{vis}(p) \mid p \in \operatorname{lnc} S\}$. By Lemma 3.1 each member is convex and compact, and by hypothesis the intersection of every $n+1$ members is not empty. Furthermore, $\operatorname{lnc} S$ is closed, hence compact. Then, Theorems 3.2 and 3.3 imply that $\operatorname{ker} S \neq \varnothing$.

THEOREM 3.7. Let $S$ be a compact connected nonconvex set in $E^{n}$ and assume that there exists $\delta>0$ such that for every $k$-pointed subset $A \subset \operatorname{lnc} S$ with $k \leqq n+1$, there is a ball $B$ of radius $\delta$ such that all the points of $B$ have higher visibility via $S$ than each of the points of $A$. Then the kernel of $S$ contains a ball of radius $\delta$.

Proof. Let $B$ be a ball of radius $\delta$ and $\mathscr{K}$ be the same family as in the previous theorem. Theorems 3.2 and 3.4 imply that ker $S$ includes a translate of $B$.

THEOREM 3.8. Let $k$ and $n$ be positive integers, $k \leqq n$, and let $h(n ; k)$ be defined by: (i) $h(n ; n)=n+1$; (ii) $h(n ; 1)=2 n$; $h(n ; k)=2 n-k$ for $n>k>1$. Let $S$ be a closed connected nonconvex set in $E^{n}$, and assume that $\operatorname{lnc} S$ is finite and such that for each m-pointed subset $A$ of $\operatorname{lnc} S$, with $m \leqq h(n ; k)$ there are $k+1$ affinely independent points having higher visibility than each of the points of $A$. Then the kernel of $S$ is of dimension at least $k$.

Proof. Consider once more the family of visibility cells of the points of local nonconvexity of $S$. The hypothesis of Theorem 3.5 holds and Theorem 3.2 implies that $\operatorname{ker} S$ has dimension at least $k$.

REMARK. Since $\operatorname{ker} S \subset \operatorname{vis}(p)$ for each $p \in S$, the hypothesis of 
Theorem 3.6 is not only sufficient but also necessary for the validity of the thesis. The same statement can be made about Theorems 3.7 and 3.8 .

\section{REFERENCES}

1. B. Grünbaum, The dimension of intersections of convex sets, Pacific J. Math., 12 (1962), 197-202.

2. E. Helly, Über Mengen konvexer Körper mit gemeinschaftlichen Punkten, Jber. Deutsch. Math. Verein., 32 (1923), 175-176.

3. V. L. Klee, Convex sets in linear spaces, Duke Math. J., 18 (1951), 443-466.

4. - The critical set of a convex body, Amer. J. Math., 75 (1953), 178-188.

5. H. Tietze, Über Konvexheit im kleinen und im grossen und über gewisse den Punkten einer Menge zugeordnete Dimensionszahlen, Math. Z., 28 (1928), 697-707.

6. F. A. Toranzos, Radial functions of convex and starshaped bodies, Amer. Math. Monthly, 74 (1967), 278-280.

7. F. A. Valentine, Local convexity and $L_{n}$ sets, Proc. of the Amer. Math. Soc., 16 (1965), 1305-1310.

Received September 3, 1980 and in revised form June 10, 1981.

Facultad de Ciencias Exactas y Naturales

Universidad de Buenos Aires

Buenos Aires, Argentina 



\section{PACIFIC JOURNAL OF MATHEMATICS}

\section{EDITORS}

DONALD BABBITT (Managing Editor)

J. DUGUNDJI

University of California

Los Angeles, California 90024

Hugo Rossi

University of Utah

Salt Lake City, UT 84112

C. C. Moore and Arthur Agus

Department of Mathematics

University of Southern California

Los Angeles, California 90007

R. FinN and J. Milgram

Stanford University

Stanford, California 94305

University of California

Berkeley, CA 94720

\section{ASSOCIATE EDITORS}
R. ARNES
E. F. BeCKenBaCH
B. H. NEUMANN
F. WoLF
K. YoshidA

\section{SUPPORTING INSTITUTIONS}

UNIVERSITY OF ARIZONA

UNIVERSITY OF BRITISH COLUMBIA

CALIFORNIA INSTITUTE OF TECHNOLOGY

UNIVERSITY OF CALIFORNIA

MONTANA STATE UNIVERSITY

UNIVERSITY OF NEVADA, RENO

NEW MEXICO STATE UNIVERSITY

OREGON STATE UNIVERSITY
UNIVERSITY OF OREGON

UNIVERSITY OF SOUTHERN CALIFORNIA

STANFORD UNIVERSITY

UNIVERSITY OF HAWAII

UNIVERSITY OF TOKYO

UNIVERSITY OF UTAH

WASHINGTON STATE UNIVERSITY

UNIVERSITY OF WASHINGTON 


\section{Pacific Journal of Mathematics}

\section{Vol. 101, No. $1 \quad$ November, 1982}

Natália Bebiano, On the evaluation of permanents $\ldots \ldots \ldots \ldots \ldots \ldots \ldots \ldots$

David Borwein and Bruce Brigham Watson, Tauberian theorems between

the logarithmic and Abel-type summability methods $\ldots \ldots \ldots \ldots \ldots \ldots 11$

Leo George Chouinard, II, Hermite semigroup rings $\ldots \ldots \ldots \ldots \ldots \ldots \ldots$

Kun-Jen Chung, Remarks on nonlinear contractions $\ldots \ldots \ldots \ldots \ldots \ldots . \ldots 4$

Lawrence Jay Corwin, Representations of division algebras over local

fields. II ......................................... 49

Mahlon M. Day, Left thick to left lumpy—a guided tour $\ldots \ldots \ldots \ldots \ldots 71$

M. Edelstein and Mo Tak Kiang, On ultimately nonexpansive

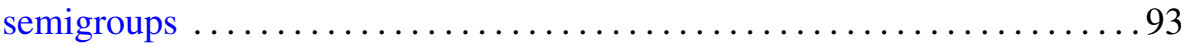

Mary Rodriguez Embry, Semigroups of quasinormal operators . ........ 103

William Goldman and Morris William Hirsch, Polynomial forms on

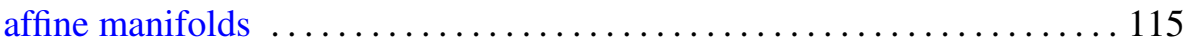

S. Janakiraman and T. Soundararajan, Totally bounded group topologies

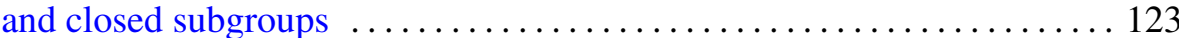

John Rowlay Martin, Lex Gerard Oversteegen and Edward D.

Tymchatyn, Fixed point set of products and cones $\ldots \ldots \ldots \ldots \ldots \ldots 133$

Jan van Mill, A homogeneous Eberlein compact space which is not metrizable ........................................ 141

Steven Paul Plotnick, Embedding homology 3-spheres in $S^{5} \ldots \ldots \ldots \ldots 147$

Norbert Riedel, Classification of the $C^{*}$-algebras associated with minimal rotations

Benedict Seifert, Combinatorial and geometric properties of weight systems of irreducible finite-dimensional representations of simple split Lie algebras over fields of 0 characteristic

James E. Simpson, Dilations on locally convex spaces

Paolo M. Soardi, Schauder bases and fixed points of nonexpansive mappings

Yoshio Tanaka, Point-countable $k$-systems and products of $k$-spaces

Fausto A. Toranzos, The points of local nonconvexity of starshaped sets . . . 209

Lorenzo Traldi, The determinantal ideals of link modules. I . . . . . . . . 215

P. C. Trombi, Invariant harmonic analysis on split rank one groups with applications

Shinji Yamashita, Nonnormal Blaschke quotients 